Check for updates

Cite this: RSC Adv., 2017, 7, 29088

\title{
Smart electrochromic supercapacitors based on highly stable transparent conductive graphene/CuS network electrodes $\uparrow$
}

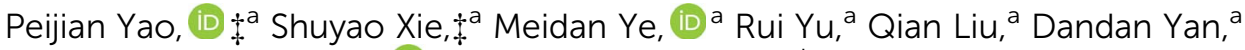 \\ Weiwei Cai, ${ }^{a}$ Wenxi Guo (D) *a and Xiang Yang Liu*ab
}

A well-designed flexible ITO-free electrochromic supercapacitor was obtained using a hybrid graphene/ CUS network on PET (G-CUS) as the transparent conductive electrode (TCE). This new type of TCE was produced by photolithography, chemical vapor deposition and a low temperature solvothermal reaction. The TCE achieves a sheet resistance $\left(R_{\mathrm{s}}\right)$ as low as $\sim 20 \Omega \mathrm{sq}^{-1}$ with a high transmittance of over $85 \%$. In particular, the G-CUS TCE displays a remarkable flexible stability under bending stresses and an excellent chemical stability through the polyaniline (PANI) electrodeposition in acid solution. The PANI/G-CuS electrode gave a favorable area capacitance of $17.3 \mathrm{mF} \mathrm{cm} \mathrm{cm}^{-2}$ at $0.025 \mathrm{~mA} \mathrm{~cm}{ }^{-2}$ along with a large optical modulation of $40.1 \%$ (620 nm). More intuitively, a symmetrical supercapacitor of PANI/G-CuS was fabricated with $\mathrm{H}_{2} \mathrm{SO}_{4}$-PVA hydrogel as the electrolyte, and its noticeable color variation enables users to evaluate the level of stored energy by the naked eye in a predictable manner. This work may provide a practical route to the fabrication of stable TCEs in smart supercapacitors.

Received 20th April 2017

Accepted 29th May 2017

DOI: $10.1039 / \mathrm{c} 7 \mathrm{ra0} 4476 \mathrm{c}$

rsc.li/rsc-advances with desired properties for applications in supercapacitors, lithium ion batteries, electrocatalysis, etc. Copper sulfide (CuS), one member of the metal sulfide family, is a well-known narrowband-gap $(\sim 2.0 \mathrm{eV})$ p-type conducting material, which has been reported by several works through directly sputtering CuS film on glass. ${ }^{29-32}$ Due to the existence of inherent trade-off between sheet resistance $\left(R_{\mathrm{s}}\right)$ and transparency of CuS films, only $60 \%$ transmittance was achieved for a $50 \mathrm{~nm}$ thickness of CuS film according to the calculation, ${ }^{30}$ and further enhancing the transmittance results in a thinner CuS layer which leads to very high $R_{\mathrm{s}}$. Despite different techniques including chemical and physical approaches have been employed to improve the quality of the CuS films, their optoelectronic performance is still poor with an optimized $R_{\mathrm{s}}$ of $\sim 100 \Omega \mathrm{sq}^{-1}$ at $\sim 75 \%$ transmittance as reported by Yuan et al. ${ }^{33}$ Therefore, it's probably the main reason why CuS is not yet applied in electrochromic supercapacitors as TCEs.

As we known, making the continuous film into mesh structure with percolating random or periodic lines is an effective strategies to transform opaque or less transparent conductors to be much transparent, in which the frameworks can conduct current and leave large empty spaces that render the film transparent. ${ }^{34,35}$ In this way, we can greatly increase the transparency of the TCEs without significantly reducing the electronic conductivity. Our group has recently reported a kind of CuS-nanosheet networks electrode with improved optoelectronic performance of $50 \Omega \mathrm{sq}^{-1}$ at $85 \%$ transmittance which was used as an efficient counter electrode for flexible quantumdot solar cell. ${ }^{36}$ However, due to the size of the spaces, those 
percolating electrodes cannot be regarded as continuous electrodes for some applications. By applying a graphene ${ }^{37}$ or PEDOT:PSS ${ }^{38}$ layer above, it is possible to make it a continuous conductive film. In addition, in the metal network electrodes, to some extent the covered PEDOT:PSS or graphene can protect the metal from oxidation. It is worth noting that besides optoelectronic performance, this design route is also beneficial for the mechanical stability of flexible and stretchable electrodes as a result of remarkable stress accommodation.

Here, we introduced a new kind of flexible TCE using CuS grids hybrid with graphene as conductive materials and PET as the supporting substrate. This graphene/CuS network (G-CuS) TCE was obtained by photolithography, chemical vapor deposition and a low temperature solvothermal reaction. It exhibits an $R_{\mathrm{s}}$ of $\sim 20 \Omega \mathrm{sq}^{-1}$ at $\sim 85 \%$ transmittance, which is superior to sputtered CuS films. In addition, the G-CuS TCE displays a remarkable mechanical flexibility and excellent chemical stability under bending stresses and polyaniline (PANI) electrodeposition in acid solution, respectively. Moreover, such symmetrical PANI supercapacitor can also act as a smart device for energy storage and its responsive color variation can simultaneously monitor the level of stored energy.

\section{Experimental section}

\section{Materials}

Ethanol (>99.7\%), sulfuric acid $\left(\mathrm{H}_{2} \mathrm{SO}_{4}, 95-98 \%\right)$ and polyvinyl alcohol (PVA-124) were purchased from Shantou Xilong chemical Co. Ltd. (Guangdong, China), and they were of analytical reagent grade (AR). Sublimed sulfur powders (AR, >99.5\%) and aniline (ACS grade, $>99.0 \%$ ) were supplied from Aladdin Co. Ltd. (Shanghai, China). All chemicals were used as received without further purification. Deionized water was used throughout the electrochemical experiments. Ag target (99.99\%) was purchased from Zhongnuoxincai (Beijing) Technology Co., Ltd.

\section{Fabrication of various flexible transparent conducting electrodes (TCEs)}

Ag networks were fabricated on PET substrates using electrospinning and sputtering methods as reported previously: ${ }^{39}$ freestanding PVA fibers were spun on an aluminium frame to form a network and then a thin layer of Ag film with a thickness of 50-100 $\mathrm{nm}$ was deposited on the polymer fiber network by magnetron sputtering. The $\mathrm{Ag}$ network was derived after transferring the whole structure onto the PET substrate and removing the PVA networks. Graphene/Cu network films $(\mathrm{G}-\mathrm{Cu})$ with $R_{\mathrm{S}}$ of $\sim 1 \Omega \mathrm{sq}^{-1}$ fabricated by photolithography and chemical vapor deposition (CVD) techniques were provided by Xiamen G-CVD Graphene Technology Co., Ltd. Then, the G-Cu films were converted into graphene/CuS network (G-CuS) TCEs by soaking them in sulfur ethanol solution $\left(0.6 \mathrm{~g} \mathrm{~L}^{-1}\right)$ at $60{ }^{\circ} \mathrm{C}$ for $12-24 h .^{40}$

\section{Electrodeposition of PANI}

The deposition of PANI was performed using a CHI 660E electrochemical workstation in conventional three-electrode system under a galvanostatic current density of $0.1 \mathrm{~mA} \mathrm{~cm} \mathrm{c}^{-2}$. One of the various TCEs was used as working electrode, a Pt foil was used as counter electrode, and an $\mathrm{Ag} / \mathrm{AgCl}$ electrode was used as reference electrode, respectively. An aqueous solution containing $0.2 \mathrm{M}$ aniline monomer and $0.5 \mathrm{M} \mathrm{H}_{2} \mathrm{SO}_{4}$ was used as the electrolyte.

\section{Assembly of a symmetrical supercapacitor}

A symmetrical supercapacitor was fabricated with $\mathrm{H}_{2} \mathrm{SO}_{4}-\mathrm{PVA}$ hydrogel as the electrolyte. ${ }^{17}$ The $\mathrm{H}_{2} \mathrm{SO}_{4}$ - $\mathrm{PVA}$ hydrogel was mixed from PVA aqueous solution and $\mathrm{H}_{2} \mathrm{SO}_{4}$ with the PVA/ $\mathrm{H}_{2} \mathrm{SO}_{4}$ mass ratio of $1 / 1$. Specifically, $5 \mathrm{~g}$ PVA was added into $45 \mathrm{~g}$ DI water and heated to $85^{\circ} \mathrm{C}$ for $4 \mathrm{~h}$ to get a clear solution. After cooling down to room temperature, $5 \mathrm{~g} \mathrm{H}_{2} \mathrm{SO}_{4}$ aqueous solution ( $85 \mathrm{wt} \%$ ) was added to the above solution under stirring for $30 \mathrm{~min}$. The $\mathrm{H}_{2} \mathrm{SO}_{4}$-PVA hydrogel electrolyte was scrapecoated on the surface of the two symmetrical G-CuS electrodes $(15 \mathrm{~mm} \times 30 \mathrm{~mm})$. Subsequently, these two electrodes were stacked face to face and the hydrogel electrolyte was used as both separator and electrolyte simultaneously. A conductive copper foil was then used to stick the edge of each electrode to form good electrical contact with an electrochemical instrument. Through this way, a symmetrical supercapacitor was attained.

\section{Characterization}

A four-point probe (RTS-8) was used to measure the films' $R_{\mathrm{s}}$. The light transmittances of all the TCEs were measured by a UVvis-NIR spectrophotometer (Perkin-Elmer Lambda 750). The morphology of the prepared materials were examined by a scanning electron microscopy (SEM, Hitachi SU-70) and a Leica microscope (DM 6000BDM 6000B). The components of G-Cu, G-CuS and G-CuS/PANI were investigated by Raman spectrometer (LabRAM HR Evolution). All electrochemical measurements were performed using a CHI660E in a conventional three-electrode system with the G-CuS/PANI, a Pt foil, an $\mathrm{Ag} / \mathrm{AgCl}$ electrode, and $0.5 \mathrm{M} \mathrm{H}_{2} \mathrm{SO}_{4}$ aqueous solution as working electrode, counter electrode, reference electrode, and the electrolyte, respectively. Areal capacitance of supercapacitors is calculated according to the following eqn (1):

$$
C=(I \times t) /(V \times S)
$$

where $C$ is areal capacitance $\left(\mathrm{F} \mathrm{cm}^{-2}\right), I$ stands for the discharge current (mA), $t$ is the discharge time (s), $V$ represents the potential window $(\mathrm{V})$, and $S$ is the effective surface area of the active material electrode $\left(\mathrm{cm}^{2}\right)$.

\section{Results and discussion}

\section{Morphology and component analysis}

Fig. 1A schematically presents the processes of the synthesis of G-CuS/PANI on polyethylene terephthalate (PET) substrate through solvothermal reaction and electropolymerization. Firstly, $\mathrm{Cu}$ networks were converted into CuS networks by immersing $\mathrm{G}-\mathrm{Cu}$ into an ethanol solution containing $0.6 \mathrm{~g} \mathrm{~L}^{-1}$ 
(A)

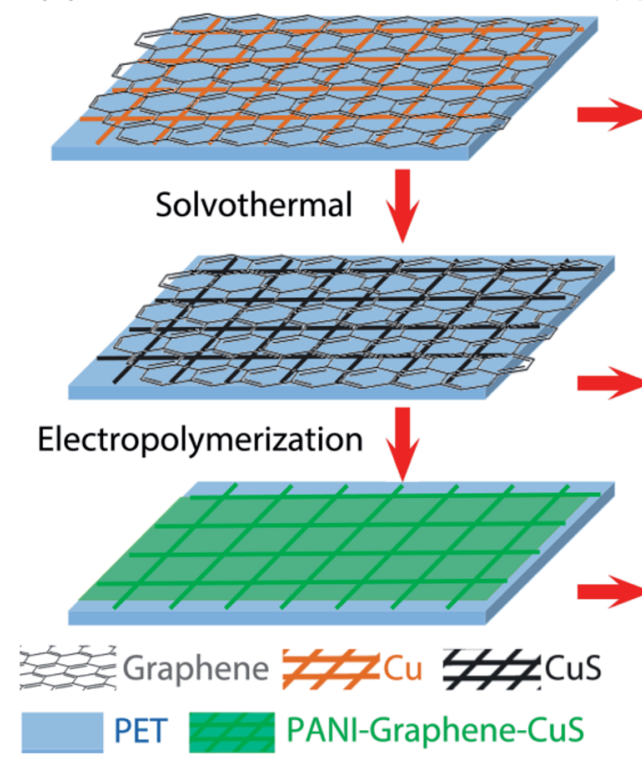

(B)

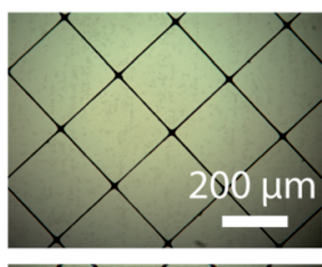

(C)

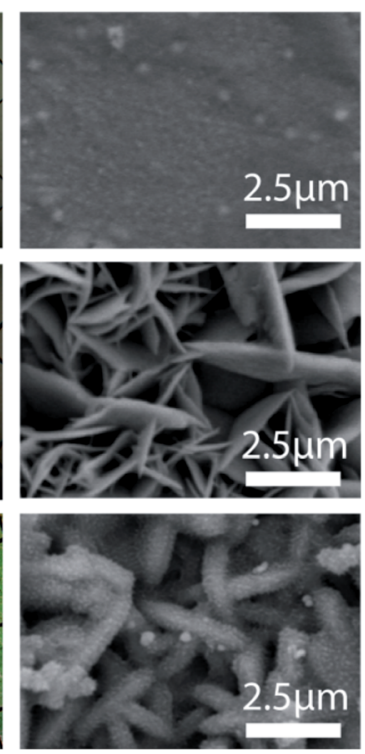

Fig. 1 (A) Schematic illustration of the processes of synthesizing G-CuS/PANI on the PET substrate through solvothermal and electropolymerization reactions. The resulted optical (B) and SEM (C) images of the corresponding film are shown on the right two columns, respectively.

sulfur. By using this simple solvothermal method, we can easily obtain a large-scale flexible G-CuS TCE (Fig. S1 in the ESI†). It follows the microscope photographs in Fig. 1B that no obvious changes were observed in the morphology after the Cu networks were converted into CuS networks. The girds of the networks have a square configuration with the $300 \mu \mathrm{m}$ length of the side $(L)$ and the $10 \mu \mathrm{m}$ width of the grid line $(w)$. Corresponding SEM morphology images shown in Fig. 1C present that smooth $\mathrm{Cu}$ grid had been transformed into sheet-like CuS grid with the sheet thickness of $\sim 50 \mathrm{~nm}$. Secondly, PANI was electrodeposited on G-CuS TCE by the electropolymerization method at a current density of $0.1 \mathrm{~mA} \mathrm{~cm}{ }^{-2}$. It follows the micrograph in Fig. 1B that the whole TCE was uniformly coated by a green film. The corresponding SEM images for the green film on grid line and empty area are displayed in Fig. 1C and 2C, respectively, indicating the whole TCE was uniformly coated by a layer of PANI nanorods arrays with a diameter of $\sim 50 \mathrm{~nm}$.

The components of G-Cu, G-CuS and G-CuS/PANI were investigated by Raman analysis in Fig. 2A. The two typical peaks of graphene located at $\sim 1580 \mathrm{~cm}^{-1}$ and $2681 \mathrm{~cm}^{-1}$ can be readily indexed to the in-plane bond stretching motion of $\mathrm{C} \mathrm{sp}^{2}$ atoms ( $\mathrm{G}$ band) and the inter-valley double resonance Raman scattering ( $\mathrm{G}^{\prime}$ band), respectively. ${ }^{41}$ In contrast to the $\mathrm{G}-\mathrm{Cu}$ networks, the Raman spectrum of the CuS nanostructures showed a distinct band at $472 \mathrm{~cm}^{-1}$, which originates from the lattice S-S stretching of covellite. ${ }^{42}$ After deposition of PANI, several weak Raman peaks located at 414-580, 1322, 1490, and $1585 \mathrm{~cm}^{-1}$ emerged in the spectrum which are attributed to the out-of-plane $\mathrm{C}-\mathrm{N}-\mathrm{C}$ torsion, $\mathrm{C}-\mathrm{N}^{+}$stretching, $\mathrm{C}=\mathrm{N}$ stretching of quinoid, and $\mathrm{C}-\mathrm{C}$ stretching of benzenoid in PANI, respectively. Furthermore, energy dispersive X-ray spectroscopy (EDS) spectrum of G-CuS/PANI is displayed in Fig. 2B. The weak peak located at $0.38 \mathrm{eV}$ is corresponding to the $\mathrm{N}$ element which belongs to PANI, which is consistent with the result of EDS mapping in Fig. 2D. Thanks to the wrapped graphene layer, PANI was not only polymerized on the surface of CuS grids, but also uniformly covered the entire G-CuS surface including the empty area as shown in Fig. 2D. On the contrary, PANI was only deposited on the CuS grids when we removed the wrapped graphene film (see Fig. S2 in the ESI $\dagger$ ). The covered graphene plays an important role to increase the electronic distribution uniformity in the electrochemical polymerization process, and it is the PANI in the empty area that dominantly causes color changes of the supercapacitor. ${ }^{38}$

\section{Physicochemical properties of TCEs}

To investigate the physicochemical properties of the G-CuS network, three other TCEs including commercial ITO, G-Cu and $\mathrm{Ag}$ networks were introduced as references. Fig. 3A shows the photograph of the four different flexible TCEs, they were commercial ITO (13 $\left.\Omega \mathrm{sq}^{-1}\right),\left(\sim 1 \Omega \mathrm{sq}^{-1}\right)$, Ag network (5 $\left.\Omega \mathrm{sq}^{-1}\right)$, and G-CuS $\left(20 \Omega \mathrm{sq}^{-1}\right)$, respectively. To examine the mechanical flexibility of the four TCEs, each sample with a size of $3.5 \mathrm{~cm} \times$ $5 \mathrm{~cm}$ was bent to the radium of $0.5 \mathrm{~cm}$, and then released to the initial position for 1000 times. As can be seen from Fig. 3B, negligible changes in the resistance were observed for $\mathrm{G}-\mathrm{Cu}, \mathrm{Ag}$ networks and G-CuS networks, while ITO suffered from serious damage and its $R_{\mathrm{S}}$ increased sharply. The broad transmittance spectra of the aforementioned four TCEs are plotted in Fig. 3C and $\mathrm{D}$. The transmittances of the Ag network, G-Cu and G-CuS are almost flat in the range of $400-2000 \mathrm{~nm}$ while that of ITO demonstrated a sharp decrease in the range of wavelength greater than $700 \mathrm{~nm}$, indicating network TCEs can solve the low 
(A)

(C)

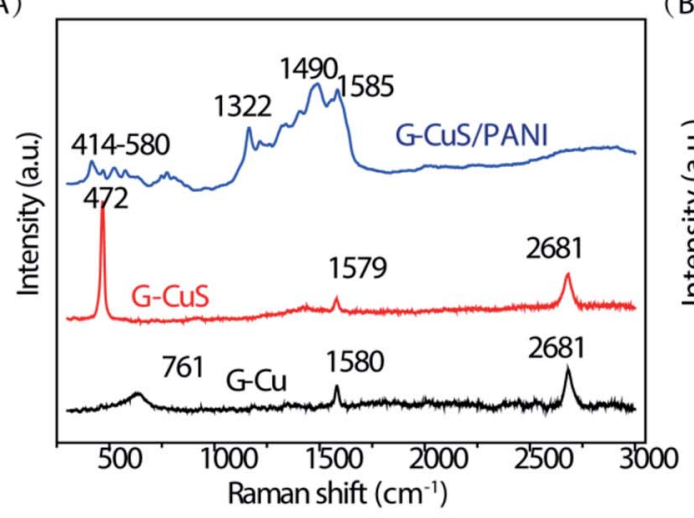

(D)
(B)



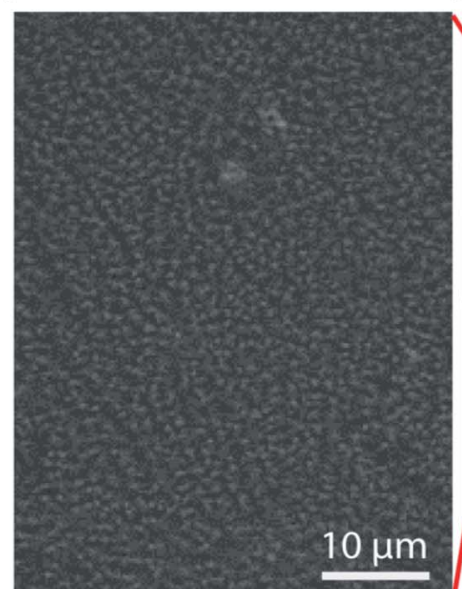
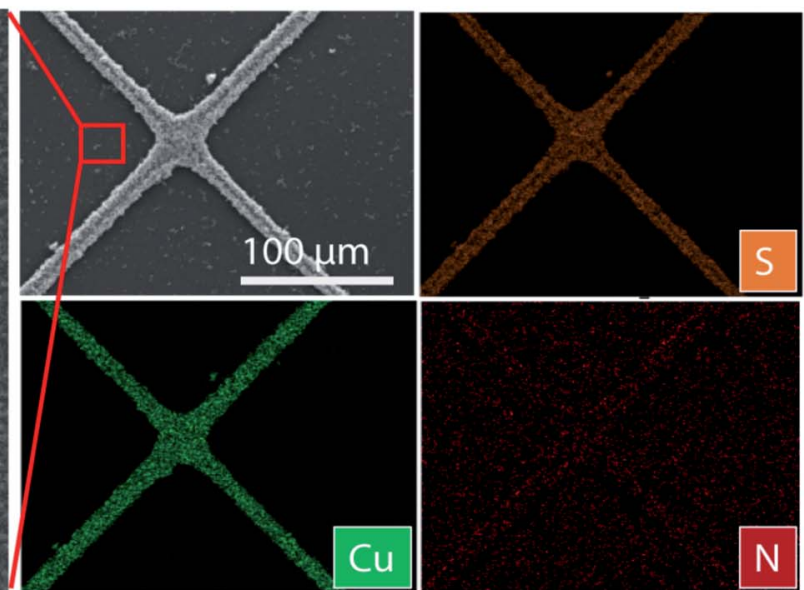

Fig. 2 (A) Raman spectra of G-Cu, G-CUS and G-CuS/PANI. (B) EDS spectrum of G-CuS/PANI. (C) SEM image of PANI grown on the G-CuS film (empty area of the grid frame). (D) EDS mapping of G-CuS/PANI demonstrates the successful fabrication of the CuS networks and the PANI film was uniformly distributed across the whole TCE.

infrared transmittance problem for the ITO films. Apart from high infrared transmittance, network TCEs exhibit excellent optoelectronic performance. The average transmittances of visible lights of Ag network $\left(5 \Omega \mathrm{sq}^{-1}\right)$ and G-Cu $\left(\sim 1 \Omega \mathrm{sq}^{-1}\right)$ are about $88.3 \%$ and $88.1 \%$, respectively, which are much better than that of ITO (13 $\Omega \mathrm{sq}^{-1}$ at a maximum transmittance of $71 \%$ ). According to the calculation from eqn (S1) and Fig. S3 in the ESI, $\dagger$ the optical transmittances of the network TCEs mainly depend upon the unpatterned area. ${ }^{35}$ Therefore, in this work, the light transmittance of the $\mathrm{Cu}$ networks should be $93.4 \%$ according to the $L$ and $w$ of the grid are $300 \mu \mathrm{m}$ and $10 \mu \mathrm{m}$, respectively. The transmittance of G-Gu TCE (88.1\%) exhibited slightly lower than that of $\mathrm{Cu}$ networks $(93.4 \%)$ can be attributed to the wrapped graphene layer reduced the light transmittance. It is worth to note that negligible decrease in transmittance was observed after converting $\mathrm{G}-\mathrm{Cu}$ networks into G-CuS networks, indicating the transparency of the CuS film can be significantly increased after making the continuous film into mesh structure.

Chemical stability is another important feature for TCEs in ECDs. Fig. 3E shows chronopotentiometric curves obtained during the galvanostatic electropolymerization of PANI on the aforementioned four flexible TCEs in an aniline- $\mathrm{H}_{2} \mathrm{SO}_{4}$ aqueous solution. For G-CuS, it reached a maximum potential value of about $0.80 \mathrm{~V}$ in less than $80 \mathrm{~s}$ corresponding to the formation of PANI, then, the potential decreased slightly and remained stable when we further extending the polymerization time. ${ }^{43}$ As for ITO, the potential remained stable in the first $880 \mathrm{~s}$ and then sharply increased due to ITO shows poor chemical stability and strong sensitivity to corrosive salts and acids. ${ }^{44}$ For Ag network and G-Cu, the deposition potentials at the beginning were only $0.42 \mathrm{~V}$ and $0.12 \mathrm{~V}$, which is attributed to their superior conductivity. However, their chemical stability was also poor and only remained stable for $20 \mathrm{~s}$ and $960 \mathrm{~s}$, respectively. It should be noted that $\mathrm{G}-\mathrm{Cu}$ exhibited higher chemical stability than $\mathrm{Ag}$ network due to the fact that the graphene can retard the metallic TCEs to some extent oxidation in erosion solution. ${ }^{20,45,46}$ Fig. $3 \mathrm{~F}$ shows the corresponding photograph before and after deposition of PANI film, indicating that PANI can be only deposited on G-CUS TCE while almost no PANI was observed on the other three TCEs. The clearer SEM images as shown in Fig. S4 (see ESI†) displays that both the Ag networks and $\mathrm{Cu}$ grids corroded and were eventually fractured. As we know, the major barrier to commercialization of most of the metallic TCE is their poor chemical stability. As for the CuS material, we has also previously demonstrated that CuS shows 
(A)

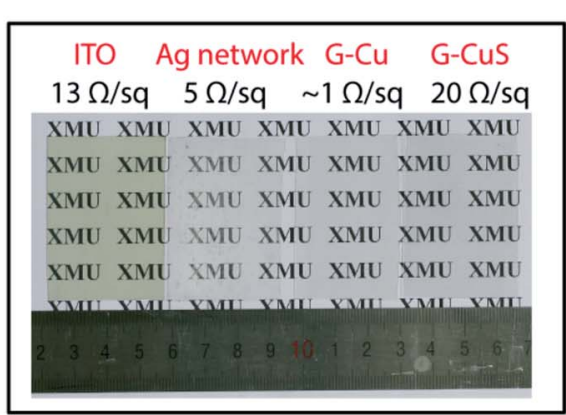

(C)



(E)



(B)

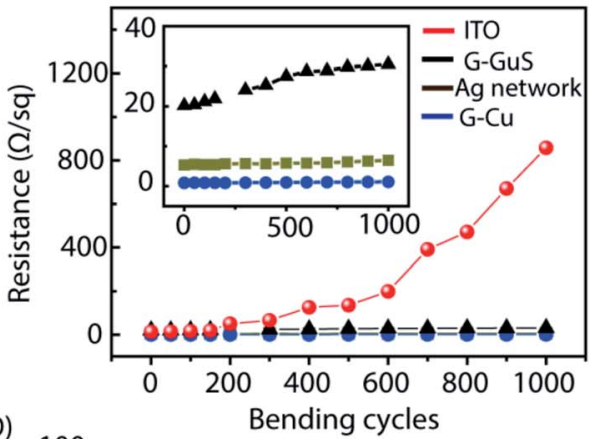

(D)

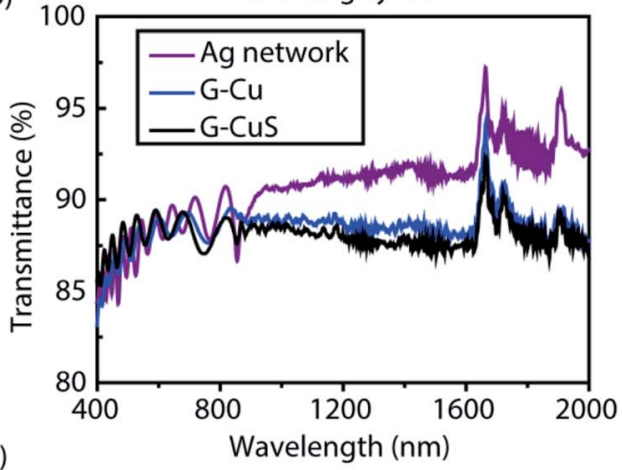

(F)

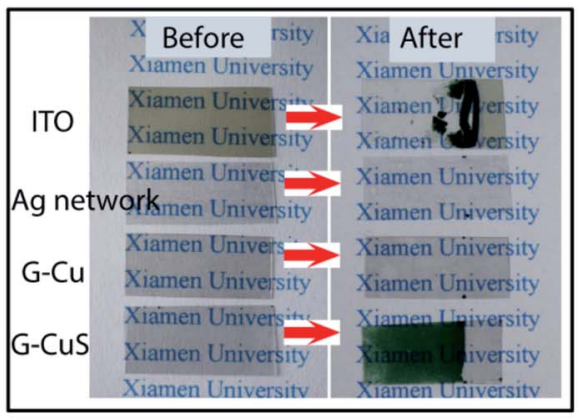

Fig. 3 (A) Photograph of the four different flexible TCEs, they are ITO, Ag networks, G-Cu and G-CuS, respectively. (B) Variations in $R_{\mathrm{s}}$ of the four different TCEs as a function of the number of bending cycles with the bending radius of $0.5 \mathrm{~cm}$. (C and D) Visible-NIR transmittance spectra of the four different TCEs. (E) Electrodeposition curves of PANI grown onto the four different TCEs under a galvanostatic density of $0.1 \mathrm{~mA} \mathrm{~cm}^{-2}$ in $0.5 \mathrm{M} \mathrm{H}_{2} \mathrm{SO}_{4}$ aqueous solution containing $0.2 \mathrm{M}$ aniline, and (F) their corresponding photos before and after electropolymerization.

excellent chemical stability when exposed to various conditions of thermal oxidation at $160{ }^{\circ} \mathrm{C}$, humid air with $80 \%$ relative humidity at $85{ }^{\circ} \mathrm{C}$, and $\mathrm{H}_{2} \mathrm{O}_{2}$ solution, respectively. ${ }^{47} \mathrm{G}-\mathrm{CuS}$ TCEs represent a new opportunity for emerging flexible electronics required working in hash conditions.

\section{Characterizations of electrochromic-supercapacitive performance}

Based on the PANI electrodeposition results, we only chose GCuS to further evaluate the possibility of its use as a TCE in the electrochromic supercapacitor. As we know, both electrochromic and supercapacitive behaviors of PANI are attributed to the redox reaction in the same acid electrolyte. ${ }^{48}$ In this context, we can simultaneously develop a multifunctional electrochromic supercapacitor that is capable of energy storage and transmittance adjustment. To evaluate the performance of the
G-CuS/PANI film as an electrochromic-supercapacitor electrode, the charge-discharge curves at different current densities (Fig. 4A) and corresponding in situ transmittance at $620 \mathrm{~nm}$ (Fig. 4B) were tested. As can be seen from Fig. 4A, both the charging and discharging time increased while decreasing charge-discharge current densities from $0.050 \mathrm{~mA} \mathrm{~cm}{ }^{-2}$ to $0.025 \mathrm{~mA} \mathrm{~cm}{ }^{-2}$. As shown in Fig. 4B, the coloring process resulted in decreasing the transmittance while the bleaching process was just the reverse. According to the Fig. $4 \mathrm{~A}$ and $\mathrm{B}$, the variation of area capacitance $(C)$ and optical modulation $(\Delta T)$ as function of current densities can be calculated and corresponding curves were displayed in Fig. 4C. It is obvious that $C$ and $\Delta T$ decreased gradually with increasing the current density. It could be attributed the fact that the migration of the electroactive ions is limited to access inner surface of the active material for energy storage owing to the diffusion effect at high current density. ${ }^{38}$ It follows from Fig. $4 \mathrm{C}$ that G-CuS/PANI 

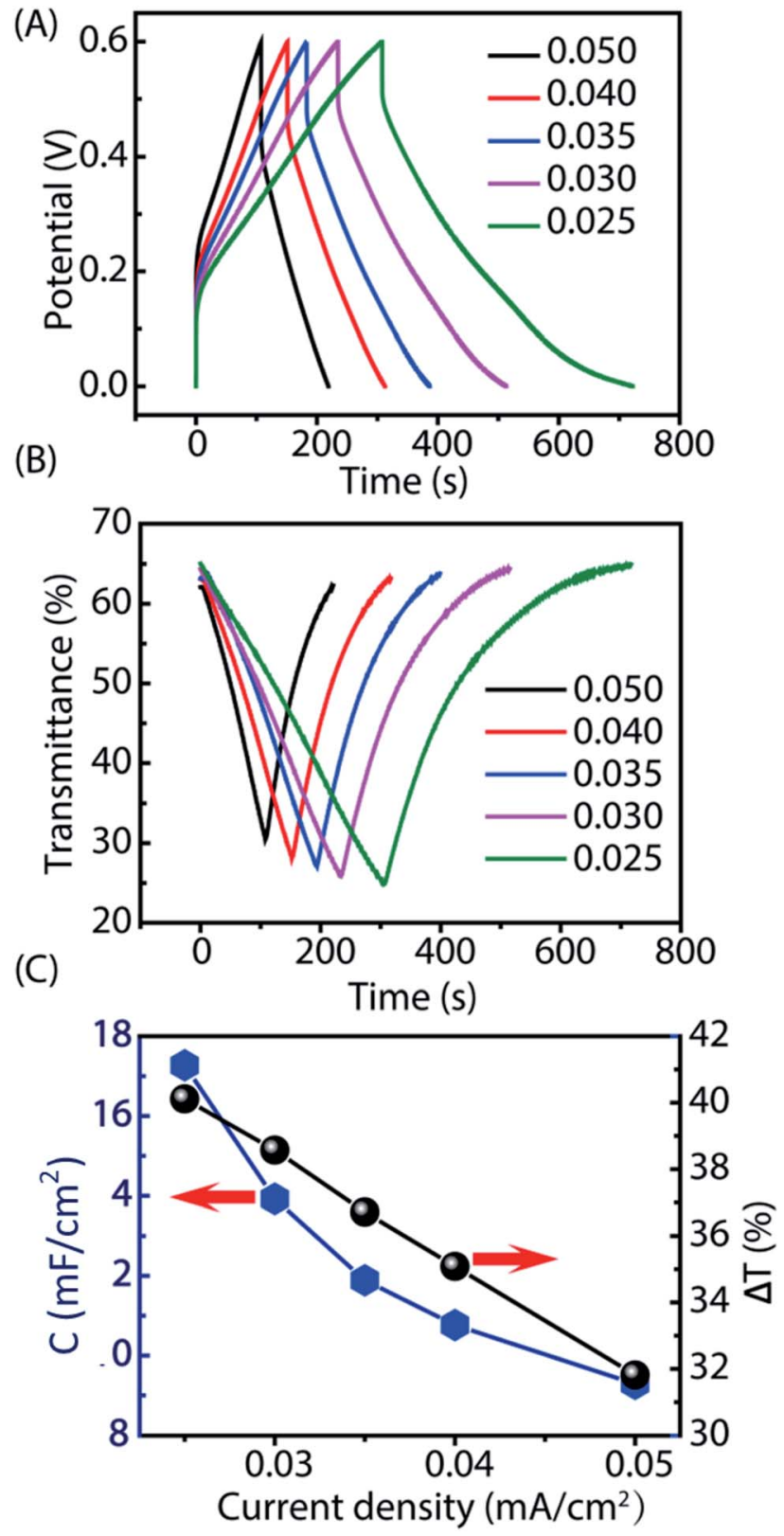

Fig. 4 Galvanostatic charge-discharge curves (A) in the potential range of 0 to $0.6 \mathrm{~V}$ and corresponding in situ optical responses (B) measured at $620 \mathrm{~nm}$ under different current densities for the G-CuS/ PANI film. (C) The dependence of area capacitance and $\Delta T$ on the current density.

exhibited area capacitances of 17.3, 13.9, 11.9, 10.8 and $9.3 \mathrm{mF}$ $\mathrm{cm}^{-2}$ at $0.025,0.030,0.035,0.040$ and $0.050 \mathrm{~mA} \mathrm{~cm} \mathrm{c}^{-2}$, along with optical modulation of $40.1 \%, 38.6 \%, 36.7 \%, 35.1 \%$ and $31.8 \%$, respectively. In addition, $C$ and $\Delta T$ at a high current density of $0.050 \mathrm{~mA} \mathrm{~cm}^{-2}$ sustained $54 \%$ and $79 \%$, respectively, compared to the values at a low current density of $0.025 \mathrm{~mA}$ $\mathrm{cm}^{-2}$. The supercapacitive results of our work is comparable to that with the PEDOT/PET as the transparent conductive electrode, which is from 6 to $15 \mathrm{mF} \mathrm{cm}$ cm $^{-2}$ when at the current density from 0.3 to $0.01 \mathrm{~mA} \mathrm{~cm}{ }^{-2} \cdot{ }^{17}$ And after galvanostatic charge-discharge tests for 200 cycles at a current density of



Fig. 5 The color change of a supercapacitor responds to the level of stored energy during the charge-discharge process (a related movie has been provided in ESI, $\uparrow$ Movie S1).

$0.045 \mathrm{~mA} \mathrm{~cm}{ }^{-2}$ as shown in Fig. S5 in the ESI, $\uparrow$ G-CuS/PANI displayed a favorable cyclic stability with a decay of only $24 \%$ compared to its initial area capacitance. Furthermore, the corresponding $I R$ drops at different current densities were displayed in Fig. S6 in the ESI. $\dagger$ The $I R$ drop is generally attributed to the charge-discharge current density besides the electrolyte potential drop and contact resistance. ${ }^{17} \mathrm{G}-\mathrm{CuS} / \mathrm{PANI}$ showed low IR drops from $76.73 \mathrm{mV}$ at $0.025 \mathrm{~mA} \mathrm{~cm}^{-2}$ to $148.87 \mathrm{mV}$ at $0.050 \mathrm{~mA} \mathrm{~cm}{ }^{-2}$. The aforementioned results demonstrate that the G-CuS/PANI electrode exhibited a supercapacitive performance and an exciting combination of the electrochromic and supercapacitive properties. Furthermore, we assembled a symmetrical supercapacitor with $\mathrm{H}_{2} \mathrm{SO}_{4}$-PVA hydrogel as the electrolyte, which presented a excellent electrochromic response to the galvanostatic charge-discharge process as shown in Fig. 5. Its noticeable color variation enables users to intuitively evaluate the level of stored energy by naked eyes in a predictable manner.

\section{Conclusions}

In summary, a newly designed G-CuS TCE with high transparency, flexibility and conductivity has been fabricated by photolithography, chemical vapor deposition and a lowtemperature solvothermal reaction. This novel TCE achieves an $R_{\mathrm{S}}$ of $\sim 20 \Omega \mathrm{sq}^{-1}$ with a transmittance of over $85 \%$. During the bending test, G-CuS exhibited remarkable mechanical stability with a small $R_{\mathrm{S}}$ increase of $\sim 10 \Omega \mathrm{sq}^{-1}$ after 1000 bending cycles. Uniform PANI was successfully deposited on the G-CuS film by the galvanostatic electrodeposition in aniline$\mathrm{H}_{2} \mathrm{SO}_{4}$ solution. The PANI/G-CuS electrode exhibited an excellent electrochromic supercapacitive performance, which achieved an area capacitance of $17.3 \mathrm{mF} \mathrm{cm} \mathrm{cm}^{-2}$ along with a large optical modulation of $40.1 \%$. Furthermore, a symmetrical supercapacitor was assembled in which the electrochromic property can intuitively reflect the condition of energy storage 
for visual assessment. The G-CuS TCE with excellent conductivity, transparency, mechanical stability and chemical stability will have a promising future in the flexible electronics. This work may supply an inspiration to the design of TCEs for electrochromic supercapacitors.

\section{Acknowledgements}

This work was supported by Beijing Natural Science Foundation (2164076), the National Nature Science Foundation of China (No. 515022553, No. U1405226), Guangdong Natural Science Foundation (2016A030310369); the Fundamental Research Funds for the Central Universities of China (No. 20720150030), the "111" Project (B16029), Fujian Provincial Bureau of Science \& Technology (2014H6022) and the 1000 Talents Program from Xiamen University.

\section{References}

1 M. Salanne, B. Rotenberg, K. Naoi, K. Kaneko, P. L. Taberna, C. P. Grey, B. Dunn and P. Simon, Nat. Energy, 2016, 1, 16070. 2 W. Li, J. Liu and D. Zhao, Nat. Rev. Mater., 2016, 1, 16023. 3 P. Simon and Y. Gogotsi, Nat. Mater., 2008, 7, 845-854.

4 P. Yang, P. Sun and W. Mai, Mater. Today, 2015, 19, 394-402. 5 G. Cai, X. Wang, M. Cui, P. Darmawan, J. Wang, A. L.-S. Eh and P. S. Lee, Nano Energy, 2015, 12, 258-267.

6 Y. Tian, S. Cong, W. Su, H. Chen, Q. Li, F. Geng and Z. Zhao, Nano Lett., 2014, 14, 2150-2156.

7 D. Wei, M. R. Scherer, C. Bower, P. Andrew, T. Ryhänen and U. Steiner, Nano Lett., 2012, 12, 1857-1862.

8 X. Chen, H. Lin, P. Chen, G. Guan, J. Deng and H. Peng, Adv. Mater., 2014, 26, 4444-4449.

9 X. Chen, H. Lin, J. Deng, Y. Zhang, X. Sun, P. Chen, X. Fang, Z. Zhang, G. Guan and H. Peng, Adv. Mater., 2014, 26, 81268132.

10 W. Liu, M. S. Song, B. Kong and Y. Cui, Adv. Mater., 2017, 29, 1603436.

11 M. Nikolou, A. L. Dyer, T. T. Steckler, E. P. Donoghue, Z. Wu, N. C. Heston, A. G. Rinzler, D. B. Tanner and J. R. Reynolds, Chem. Mater., 2009, 21, 5539-5547.

12 L. Hu, G. Gruner, D. Li, R. B. Kaner and J. Cech, J. Appl. Phys., 2007, 101, 016102.

13 J. H. Lee, D. W. Shin, V. G. Makotchenko, A. S. Nazarov, V. E. Fedorov, Y. H. Kim, J.-Y. Choi, J. M. Kim and J.-B. Yoo, Adv. Mater., 2009, 21, 4383-4387.

14 W. R. Lian, Y. C. Huang, Y. A. Liao, K. L. Wang, L. J. Li, C. Y. Su, D. J. Liaw, K. R. Lee and J. Y. Lai, Macromolecules, 2011, 44, 9550-9555.

15 C. Sachse, N. Weiß, N. Gaponik, L. Müller-Meskamp, A. Eychmüller and K. Leo, Adv. Energy Mater., 2014, 4, 1300737.

16 C. Lee, C. Kim, M. Jeong, J. Kim, J. Lee, J. W. Oh, J. Lee, S. H. Kim, S. S. Park and J. M. Kim, $R S C$ Adv., 2015, 5, 77288-77295.

17 K. Wang, H. Wu, Y. Meng, Y. Zhang and Z. Wei, Energy Environ. Sci., 2012, 5, 8384-8389.
18 K. Sanglee, S. Chuangchote, P. Chaiwiwatworakul and P. Kumnorkaew, J. Nanomater., 2017, 2017, 8.

19 B. W. An, B. G. Hyun, S.-Y. Kim, M. Kim, M.-S. Lee, K. Lee, J. B. Koo, H. Y. Chu, B.-S. Bae and J.-U. Park, Nano Lett., 2014, 14, 6322-6328.

20 Y. Ahn, Y. Jeong, D. Lee and Y. Lee, ACS Nano, 2015, 9, 31253133.

21 Y. H. Kim, L. Müller-Meskamp and K. Leo, Adv. Energy Mater., 2015, 5, 1401822.

22 S. K. Balasingam, M. Lee, B. H. Kim, J. S. Lee and Y. Jun, Dalton Trans., 2017, 46, 2122-2128.

23 X. Li, W. Li, M. Li, P. Cui, D. Chen, T. Gengenbach, L. Chu, H. Liu and G. Song, J. Mater. Chem. A, 2015, 3, 2762-2769.

24 B. M. Gray, A. L. Hector, M. Jura, J. R. Owen and J. Whittam, J. Mater. Chem. A, 2017, 5, 4550-4559.

25 W. Li, C. Y. Cao, C. Q. Chen, Y. Zhao, W. G. Song and L. Jiang, Chem. Commun., 2011, 47, 3619-3621.

26 W. Li, X. Gao, D. Xiong, F. Xia, J. Liu, W. G. Song, J. Xu, S. M. Thalluri, M. F. Cerqueira, X. Fu and L. Liu, Chem. Sci., 2017, 8, 2952-2958.

27 Z. Zhang, S. Liu, J. Xiao and S. Wang, J. Mater. Chem. A, 2016, 4, 9691-9699.

28 W. Li, X. Gao, X. Wang, D. Xiong, P.-P. Huang, W.-G. Song, X. Bao and L. Liu, J. Power Sources, 2016, 330, 156-166.

29 A. Cuevas, R. Romero, D. Leinen, E. A. Dalchiele, J. R. RamosBarrado and F. Martin, Sol. Energy Mater. Sol. Cells, 2015, 134, 199-208.

30 S. V. Bagul, S. D. Chavhan and R. Sharma, J. Phys. Chem. Solids, 2007, 68, 1623-1629.

31 P. Parreira, G. Lavareda, J. Valente, F. T. Nunes, A. Amaral and C. N. de Carvalho, Phys. Status Solidi A, 2010, 207, 1652-1654.

32 P. Parreira, G. Lavareda, A. Amaral, A. M. Botelho do Rego, O. Conde, J. Valente, F. Nunes and C. Nunes de Carvalho, J. Alloys Compd., 2011, 509, 5099-5104.

33 K. D. Yuan, J. J. Wu, M. L. Liu, L. L. Zhang, F. F. Xu, L. D. Chen and F. Q. Huang, Appl. Phys. Lett., 2008, 93, 132106.

34 N. Fukaya, D. Y. Kim, S. Kishimoto, S. Noda and Y. Ohno, ACS Nano, 2014, 8, 3285-3293.

35 B. Y. Ahn, D. J. Lorang and J. A. Lewis, Nanoscale, 2011, 3, 2700-2702.

36 Z. Xu, T. Li, F. Zhang, X. Hong, S. Xie, M. Ye, W. Guo and X. Liu, Nanoscale, 2017, 9, 3826-3833.

37 Y. Zhu, Z. Sun, Z. Yan, Z. Jin and J. M. Tour, ACS Nano, 2011, 5, 6472-6479.

38 G. Cai, P. Darmawan, M. Cui, J. Wang, J. Chen, S. Magdassi and P. S. Lee, Adv. Energy Mater., 2016, 6, 1501882.

39 X. M. Wu, W. Z. Zhang, Q. G. Wang, Y. Wang, H. Y. Yan and W. X. Chen, Synth. Met., 2016, 212, 1-11.

40 W. Ke, G. Fang, H. Lei, P. Qin, H. Tao, W. Zeng, J. Wang and X. Zhao, J. Power Sources, 2014, 248, 809-815.

41 L. M. Malard, M. A. Pimenta, G. Dresselhaus and M. S. Dresselhaus, Phys. Rep., 2009, 473, 51-87.

42 Y. Du, Z. Yin, J. Zhu, X. Huang, X.-J. Wu, Z. Zeng, Q. Yan and H. Zhang, Nat. Commun., 2012, 3, 1177.

43 J. Yang and D. C. Martin, Sens. Actuators, B, 2004, 101, 133142. 
44 S. W. Gaarenstroom, M. P. Balogh, M. C. Militello, R. A. Waldo, C. A. Wong, N. A. Kelly, T. L. Gibson and M. D. Kundrat, Surf. Interface Anal., 2005, 37, 385-392.

45 W. Wu, N. G. Tassi, D. J. Walls, L. Zhang and B. Willner, Appl. Phys. Lett., 2014, 105, 223104.
46 R. Mehta, S. Chugh and Z. Chen, Nano Lett., 2015, 15, 20242030.

47 X. Zhang, W. Guo, G. Gao, M. Que, C. Pan and Z. L. Wang, J. Mater. Chem. C, 2016, 4, 4733-4739.

48 X. Xiang, W. Zhang, Z. Yang, Y. Zhang, H. Zhang, H. Zhang, H. Guo, X. Zhang and Q. Li, RSC Adv., 2016, 6, 24946-24951. 